\title{
Persistent Respiratory Irregularity in Patients with Panic Disorder
}

\author{
James L. Abelson, John G. Weg, Randolph M. Nesse, and George C. Curtis
}

\begin{abstract}
Background: Dysregulated respiratory control may play a role in the pathophysiology of panic disorder. This could be due to abnormalities in brain stem respiratory nuclei or to dysregulation at higher brain levels. Results from previous studies using the doxapram model of panic have yielded an unclear picture. A brief cognitive manipulation reduced doxapram-induced hyperventilation in patients, suggesting that higher level inputs can substantially alter their respiratory patterns. However, respiratory abnormalities persisted, including a striking irregularity in breathing patterns.
\end{abstract}

Methods: To directly study respiratory irregularity, breath-by-breath records of tidal volume $\left(V_{t}\right)$ and frequency (f) from previously studied subjects were obtained. Irregularity was quantified using von Neumann's statistic and calculation of "sigh" frequency in 16 patients and 16 matched control subjects. Half of each group received a standard introduction to the study and half received a cognitive intervention designed to reduce anxiety/distress responses to the doxapram injection.

Results: Patients had significantly greater $V_{t}$ irregularity relative to control subjects. Neither the cognitive intervention nor doxapram-induced hyperventilation produced significant changes in $V_{t}$ irregularity. The $V_{t}$ irregularity was attributable to a sighing pattern of breathing that was characteristic of panic patients but not control subjects. Patients also had somewhat elevated firregularity relative to control subjects.

Conclusions: The irregular breathing patterns in panic patients appear to be intrinsic and stable, uninfluenced by induced hyperventilation or cognitive manipulation. Further study of $V_{t}$ irregularity and sighs are warranted in efforts to localize dysregulated neural circuits in panic to brain stem or midbrain levels. Biol Psychiatry 2001;49: 588-595 ㅇ 2001 Society of Biological Psychiatry

Key Words: Panic disorder, respiratory irregularity, sighs, doxapram

\footnotetext{
From Anxiety Disorders Program, Department of Psychiatry (JLA, RMN, GCC) and Pulmonary and Critical Care Medicine Division, Department of Internal Medicine (JGW), University of Michigan, Ann Arbor.

Address reprint requests to James L. Abelson, M.D., Ph.D., Program Director \& Associate Professor, University of Michigan, Department of Psychiatry, Anxiety Disorders Program, 1500 E. Medical Center Drive, Room C435, Med Inn Bldg./0840, Ann Arbor MI 48109-0840.

Received September 6, 2000; accepted October 11, 2000.
}

\section{Introduction}

$\mathrm{C}$ onsiderable evidence now supports a hypothesized brain stem, respiratory control center abnormality in patients with panic disorder-described as a hypersensitive suffocation alarm system (Klein 1993). Panic patients are more sensitive to the anxiogenic effects of $\mathrm{CO}_{2}$ inhalation, and patients who panic in response to $\mathrm{CO}_{2}$ demonstrate several respiratory abnormalities (Papp et al 1997). Panic patients also display excessive behavioral and respiratory responses to the respiratory stimulant doxapram (Abelson et al 1996a; Lee et al 1993). Respiratory abnormalities have been detected in panic patients even during sleep (Stein et al 1995). Effective antipanic medications have respiratory effects that may mediate their clinical impact (Papp et al 1993).

Some recent work, however, has failed to lend unequivocal support for the hypothesis of a specific, dysregulated suffocation alarm system in panic. For example, Roth et al (1998) predicted that an overly sensitive suffocation alarm should result in excessive psychologic and physiologic responses to breath holding, but they failed to find such excessive responses in their panic patients. A competing explanation of available data has suggested that respiratory hypersensitivity in panic may be secondary to cognitive abnormalities (Clark 1993). Patients with panic disorder may differ from normal subjects not in any specific biological sensitivity, but in a cognitive set that interprets many bodily sensations as potentially harmful. According to this model, respiratory dysregulation may be secondary to dysregulated cognitive and emotional processes, rather than generative of them. Demonstrations that cognitive manipulations can attenuate panic responses to a number of agents (Albus et al 1992; Clark 1993; Rapee et al 1986; Sanderson et al 1989; van der Molen et al 1986) have been offered as support for this hypothesis.

In a laboratory model of panic that uses the respiratory stimulant doxapram, panic patients have shown a dramatically exaggerated hyperventilatory response relative to control subjects (Abelson et al 1996a; Lee et al 1993), reflected in elevated respiratory frequency $(f)$ and minute ventilation $\left(\mathrm{V}_{\mathrm{E}}\right)$ and depressed end tidal partial pressure of carbon dioxide $\left(\mathrm{pCO}_{2}\right)$. A brief cognitive intervention, 
designed to reduce panic responses, greatly reduced the excessive $f, \mathrm{~V}_{\mathrm{E}}$, and depression of $\mathrm{pCO}_{2}$ seen in panic patients. It had little effect on tidal volume $\left(\mathrm{V}_{\mathrm{t}}\right)$. Inspection of breathing records suggested that the breathing of panic patients was never fully "normalized," despite the generally normalizing impact of the cognitive intervention, and that what persisted was an irregularity in their breathing patterns. Other investigators have noted irregular breathing in panic patients. These patients show an increased SD of $\mathrm{V}_{\mathrm{t}}$ before $\mathrm{CO}_{2}$ inhalation (Gorman et al 1988), frequent and irregular respiratory pauses preceding and accompanying $\mathrm{CO}_{2}$-induced panic (Bystritsky and Shapiro 1992), and $\mathrm{V}_{\mathrm{t}}$ irregularity that persists even during sleep (Stein et al 1995). Irregularity in breathing patterns, perhaps due to a high frequency of sighs (Schwartz et al 1996), could provide a stable marker of dysregulation within respiratory control centers of panic patients. To explore this possibility further, we re-examined the effects of the cognitive intervention in our doxapram study. Breath-by-breath records were used to look specifically at irregularity of $\mathrm{V}_{\mathrm{t}}$ and at sighs, at rest and in response to doxapram. If respiratory irregularity in panic patients reflects intrinsic dysregulation affecting brain respiratory control centers, elevations should be seen in patients throughout all phases of the experiment and should be unaffected by cognitive intervention.

\section{Methods and Materials}

The subjects and experimental procedures were described in detail previously (Abelson et al 1996a). Briefly, sixteen medically healthy, medication-free patients with DSM-III-R panic disorder with or without agoraphobia were compared to16 ageand gender-matched healthy control subjects. All provided written informed consent. Subjects were studied in a single, threephase experimental session that included a 5-min accommodation period, a 15 -min placebo injection phase ( $5 \mathrm{~min}$ baseline and 10 min postplacebo monitoring), and a 30-min doxapram injection phase ( $5 \mathrm{~min}$ baseline and $25 \mathrm{~min}$ postdoxapram monitoring). The phases were separated by a 5-min structured interview to elicit symptom ratings and description of experiences. Subjects were told that the first period was for acclimatization only and that in the subsequent phases they might receive two placebo injections, two doxapram injections, or one of each in either order. However, all received the placebo first to preserve the blind, since doxapram's effects make its blind administration impossible. Patients were randomly assigned to either a "standard" or an "experimental" condition. The standard condition used a routine factual, prestudy introduction/orientation. The experimental condition applied a cognitive intervention designed to reduce subjective anxiety and panic responses (see below). Control subjects received the same introduction as their paired patients. The study thus had four cells, with diagnosis (patients vs. control subjects) crossed with cognitive set (standard vs. experimental). The experimenter who conducted the symptom interview was blind to instruction group assignment.
Subjects reported to the clinic for study at 1:30 PM. They received either the standard introduction or the cognitive intervention via audiotape. During study they sat in a reclining chair with backs to investigators and an intravenous catheter in place in a forearm or antecubital vein. A mouthpiece and nose clip were put in place just as each phase was initiated. Respiratory data were collected via a CAD*NET SYSTEM 2001 (Medical Graphics Corp., St. Paul), which provides a continuous, breathby-breath recording of end tidal $\mathrm{pCO}_{2}, \mathrm{~V}_{\mathrm{E}}, f$, and $\mathrm{V}_{\mathrm{t}}$. The placebo injection consisted of $5 \mathrm{~mL}$ of normal saline. The doxapram was given in a dose of $0.5 \mathrm{mg} / \mathrm{kg}$, in a $5-\mathrm{mL}$ solution with normal saline. Both were injected over $15 \mathrm{sec}$, outside of the subject's field of vision.

The standard introduction paralleled the control instructions used in earlier studies of cognitive factors in laboratory models of panic (Clark 1993; Sanderson et al 1989). It fully described the apparatus, procedures, and common side effects of doxapram via a 4-min tape recording. The cognitive intervention consisted of an inoculation against "catastrophic misinterpretation" of doxapram side effects and an illusion of control over the doxapram administration. It included the following components: 1) more detailed information (on tape) of expectable responses, with coaching to attribute these responses to normal reactions to doxapram rather than anything dangerous; 2) a brief verbal exploration of the subject's own experience with somatic sensations as anxiety triggers to illustrate how "normal" sensations could become cues for panic; and 3) provision of an "illusion of control" by informing subjects (falsely) that they could control their exposure to doxapram (if they needed to) using an infusion pump at their side.

\section{Analyses}

We quantified irregularity of $\mathrm{V}_{\mathrm{t}}$ and $f$ using the von Neumann statistic, calculated over 5-min blocks for each phase (one block in the accommodation phase, three blocks in the placebo phase, and six blocks in the doxapram phase). This statistic is the sum of squared differences between successive breaths, divided by the number of differences summed. We utilized the square root of the von Neumann statistic in subsequent analyses.

The central questions were whether patients and controls differed in $\mathrm{V}_{\mathrm{t}}$ or $f$ irregularity, and whether cognitive set or experimental phase had any impact on irregularity or on patientcontrol subject differences in irregularity. To address these questions the means of the square roots of von Neumann statistics for each phase were used in "diagnosis by cognitive set by phase" analyses of variance (ANOVAs), with phase as a within-subject variable. We also more directly examined the impact of doxapram-induced hyperventilation on breathing irregularity by comparing postdoxapram $\mathrm{V}_{\mathrm{t}}$ and $f$ irregularity measures to predoxapram baselines, using paired $t$ tests.

To assess the contribution of a sighing pattern of breathing to detected irregularity, we quantitatively defined a sigh as any breath that was at least $500 \mathrm{~mL}$ larger than the mean of the prior three breaths and at least $400 \mathrm{~mL}$ larger than the following breath. When two successive breaths were both $500 \mathrm{~mL}$ larger than the mean of the three breaths preceding the first and at least $400 \mathrm{~mL}$ larger than the breath following the second, both were 

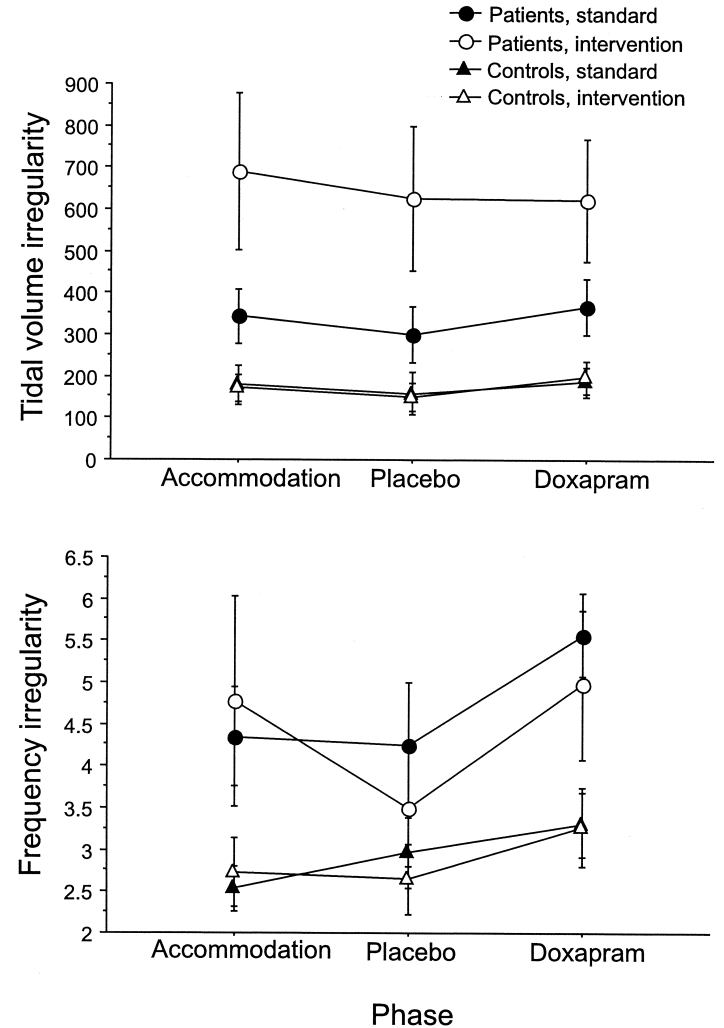

Figure 1. Tidal volume and frequency irregularity in four subject groups (patients and control subjects, each receiving either a standard introduction or a cognitive intervention before the study session). Irregularity is measured by the von Neumann statistic (see Methods and Materials), calculated over 5-min blocks and then averaged across blocks within the three experimental phases (shown \pm SEs).

counted. When there were three or more successive such breaths they were considered a hyperventilatory run and not counted as sighs. Patients and control subjects were compared on rate of sighs (per 5-min block) in a "diagnosis by cognitive set by time" ANOVA. This analysis used three time points-accommodation phase, predoxapram baseline, and postdoxapram (mean of the five postinjection blocks). We used the predoxapram baseline instead of placebo phase values, as we were particularly interested in whether sigh rate changed from pre- to postdoxapram. However, sigh rates were also counted during the placebo phase and were essentially identical to those seen during accommodation and predoxapram baseline. Including them added nothing to the analyses, and they are not reported here.

\section{Results}

Group means for $\mathrm{V}_{\mathrm{t}}$ irregularity during the three experimental phases are presented in Figure 1. Patients had significantly greater $\mathrm{V}_{\mathrm{t}}$ irregularity than did control subjects $[F(1,28)=12.64, p=.001]$. Alhough it appears that patients who received the cognitive intervention may have had higher $\mathrm{V}_{\mathrm{t}}$ irregularity than those who did not, neither the main effect of cognitive set nor the diagnosis by cognitive set interaction reached significance $[F(1,28)=$ $2.95, p=.10 ; F(1,28)=3.03, p=.09$, respectively]. There were no changes in $\mathrm{V}_{\mathrm{t}}$ irregularity between accommodation, placebo, and doxapram phases $[F(2,56)=1.09$, $p=.34$, suggesting that this measure did not change over time and was unaffected by the profound hyperventilation induced by doxapram.

Group means for $f$ irregularity during the three phases are also presented in Figure 1. Patients had significantly greater $f$ irregularity than did control subjects $[F(1,28)=$ 9.58, $p=.004]$. Cognitive set had no impact on $f$ irregularity $[F(1,28)=0.11, p=.74]$. There was a significant effect of phase $[F(2,56)=4.98, p=.01]$, due primarily to increased $f$ irregularity in the doxapram phase.

The above ANOVAs suggest that doxapram, which produced hyperventilation by increasing both $\mathrm{V}_{\mathrm{t}}$ and $f$ (Abelson et al 1996a), also increased irregularity in breathing frequency but did not increase irregularity of $\mathrm{V}_{\mathrm{t}}$. Paired $t$ tests combining all subjects (patients and control subjects) confirmed this impression. Postdoxapram $f$ irregularity was significantly greater than the predoxapram baseline $[t(31)=2.75, p=.01]$. Tidal volume irregularity did not differ from pre- to postdoxapram $[t(31)=0.66$, $p=.52]$. These findings held true even when comparing the $5 \mathrm{~min}$ immediately following doxapram, when hyperventilation was most profound, to the predoxapram baseline $\left[f\right.$ irregularity, $t(31)=2.73, p=.01 ; \mathrm{V}_{\mathrm{t}}$ irregularity, $t(31)=0.33, p=.74]$.

The irregularity of breathing patterns in panic patients was most visually evident in breath-by-breath graphs of $\mathrm{V}_{\mathrm{t}}$. Healthy subjects tended to show little $\mathrm{V}_{\mathrm{t}}$ variation between breaths, whereas panic patients tended to show greater variation, dominated by their more frequent, prominent sighs. Illustrative individual subject records that highlight the described differences are presented in Figure 2. The ANOVAs on sigh data confirm the excessive sighing seen in patients' $V_{t}$ graphs (data presented in Figure 3). Patients had a significantly greater rate of sighs than control subjects $[F(1,28)=13.73, p=.0009]$. Doxapram-induced hyperventilation had no impact on sigh rates (Figure 3) [no effect of time, $F(2,56)=0.07$, $p=.93$ ] , and the elevated rates in patients persisted at all time points (no significant interactions involving diagnosis, $p>.55)$. The cognitive intervention had no impact on sigh rates $[F(1,28)=0.27, p=.61]$, and the elevated rates in patients were not impacted by cognitive set (no significant interactions, $p>.55$ ). To contrast the stability of sigh frequency with the significant changes in overall ventilation due to doxapram and the cognitive intervention, we reanalyzed $V_{E}$ data for the identical time periods included in the sigh frequency ANOVA. These data are also included in Figure 3. Patients had greater $V_{E}$ than 

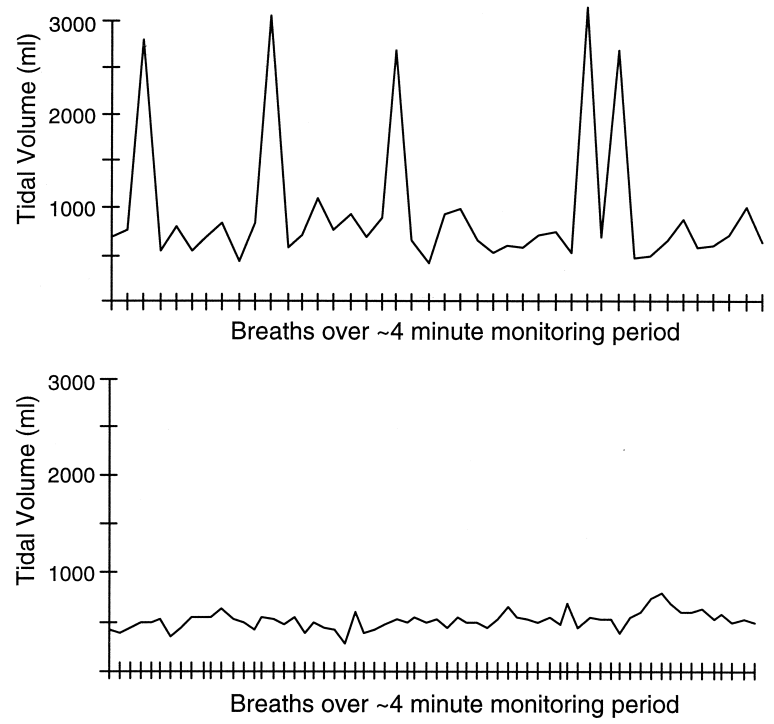

Figure 2. Graphs of tidal volume records for two subjects from the accommodation phase. Each mark on the $\mathrm{x}$ axis represents a single breath, and each breath's tidal volume is shown on the y axis. The total monitoring period depicted was slightly less than 4 min. (Top) From a panic disorder patient whose tidal volume pattern shows slight basal irregularity, punctuated by five very large tidal volume breaths, or sighs. (Bottom) From a healthy control subject who shows a highly stable tidal volume pattern, typical of most control subjects.

control subjects in all phases $[F(1,28)=8.48, p=.007]$, but in contrast to sigh frequency, $\mathrm{V}_{\mathrm{E}}$ increased significantly in response to doxapram $[F(2,56)=17.45, p<$ $.0001]$ and did so to a greater degree in patients than in control subjects [diagnosis by time interaction, $F(2,56)=$ $4.86, p=.01]$. Also in contrast to sigh frequency, $\mathrm{V}_{\mathrm{E}}$ response to doxapram was altered by the cognitive intervention [instruction by time interaction, $F(2,56)=3.47$, $p=.04$ ], due to its large impact on patients [diagnosis by instruction by time interaction, $F(2,56)=3.52, p=.04]$.

Sighs, as defined in Analyses, accounted for a substantial portion of the $V_{t}$ irregularity captured by the von Neumann statistic. The total number of sighs during the accommodation and doxapram phases was highly correlated with the mean $\mathrm{V}_{\mathrm{t}}$ irregularity during these phases, measured by the von Neumann statistic $(r=.84, p<$ $.0001, n=32$ ).

Using a definition of sighs based on a fixed breath size for all subjects can introduce bias if subjects differed systematically in basal $\mathrm{V}_{\mathrm{t}}$. We explored the possibility of such bias in our data in two ways. We compared groups, using the group by cognitive set ANOVA, on tidal volume - with sighs deleted - during the accommodation phase. We found no effect of diagnosis $[F(1,28)=2.20$, $p=.15]$ or cognitive set $[F(1,28)=0.19, p=.67]$ and no interaction $[F(1,28)=0.79, p=.38]$, indicating that there were no group differences in the best available measure of "basal" $\mathrm{V}_{\mathrm{t}}$. We also recalculated sigh frequency during accommodation phase using a sigh definition that adjusted the fixed criteria (500 $\mathrm{mL}$ increase and $400 \mathrm{~mL}$ decrease) by a factor reflecting the deviation of a subject at baseline from the pooled sample mean. The adjustment factor used was each subject's mean nonsigh $V_{t}$ during accommodation phase divided by the pooled group mean for this variable. Sigh frequency during the accommodation phase was recounted using these individualized criteria. This had no real impact on the results. Four sighs were lost and one gained, for a net change of three out of a total of 43 sighs. The elevation of sigh frequency in panic patients remained highly significant $(F=8.29, p=.008$ using the individualized definition; $F=8.89, p=.006$ using the original definition).

\section{Discussion}

Initial analyses of data from this experiment (Abelson et al 1996a) used the monitoring system's usual output of
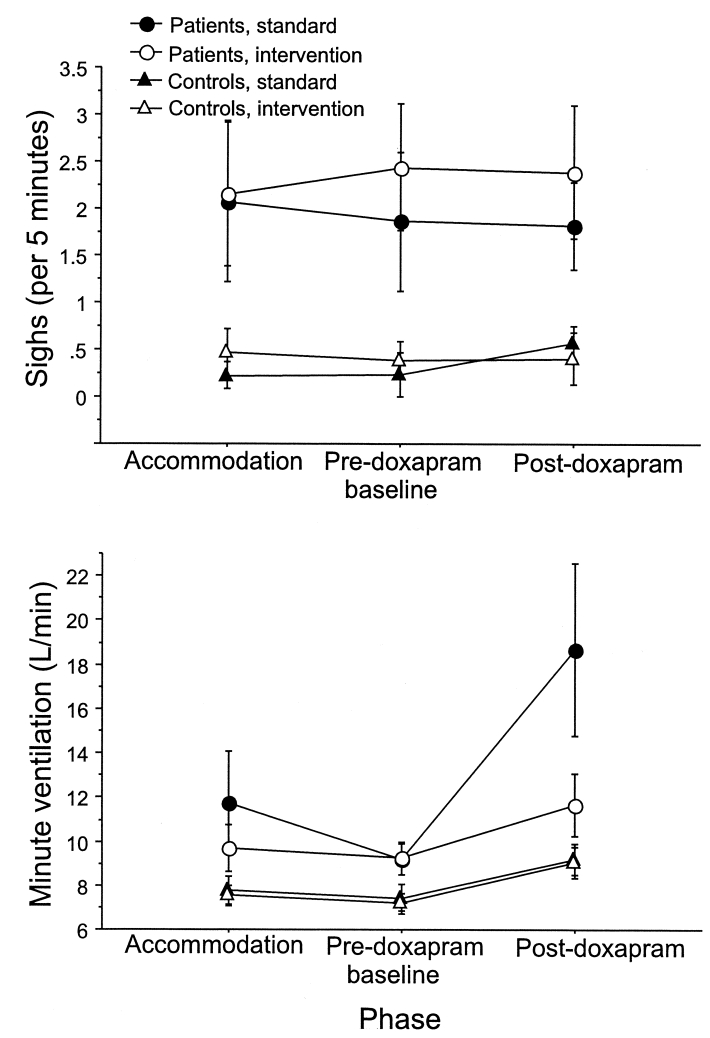

Figure 3. (Top) The number of sighs per $5 \mathrm{~min}$ of monitoring (means \pm SEs) in the four subject groups (patients and control subjects, each receiving either a standard introduction or a cognitive intervention). The accommodation phase was 5 min long. The predoxapram baseline was a 5-min period of rest before administration of doxapram. For the postdoxapram phase, sighs per 5-min block were averaged over the five blocks $(25$ min) of this phase. (Bottom) Allows comparison to minute ventilation over the same time periods. 
1-min mean data to show that doxapram increased respiratory rate, $V_{t}$, and $V_{E}$ and markedly reduced end tidal $\mathrm{pCO}_{2}$. The changes in rate, $\mathrm{V}_{\mathrm{E}}$, and $\mathrm{pCO}_{2}$ were significantly greater in panic disorder patients relative to control subjects, and a higher proportion of patients experienced panic attacks. However, a brief cognitive intervention, which was designed to minimize catastrophic misinterpretation of somatic cues and to provide an "illusion of control," substantially normalized patients' respiratory responses and reduced rates of panic. These findings could be interpreted as support for the catastrophic cognitive misinterpretation hypothesis of panic. They also support the possibility that some respiratory abnormalities in panic could be secondary to activity in components of the anxiety circuitry outside of brain stem respiratory control centers. However, the cognitive intervention had little impact on $\mathrm{V}_{\mathrm{t}}$, which appeared unstable in panic patients regardless of cognitive state. The possibility remained of a fundamental respiratory dysregulation, reflected in unstable breathing patterns, which would be consistent with Klein's suffocation alarm hypothesis (Klein 1993).

Our analyses used breath-by-breath records to confirm and extend the prior impression of instability in the respiratory patterns of panic patients. Panic patients showed abnormal irregularity of both $\mathrm{V}_{\mathrm{t}}$ and $f$. The $f$ irregularity was somewhat state sensitive, changing with time and with doxapram-induced hyperventilation, whereas $V_{t}$ irregularity showed little change. The persistent $\mathrm{V}_{\mathrm{t}}$ irregularity in panic patients was primarily attributable to a breathing pattern characterized by frequent sighs. This pattern also showed little change over time despite substantial, doxapram-induced hyperventilation. It was also uninfluenced by a cognitive intervention that reduced panic responses (Abelson et al 1996a). Despite substantial and significant differences over time in $\mathrm{V}_{\mathrm{E}}$ and in subjective anxiety and panic symptoms, sigh frequency remained relatively stable and persistently elevated, both within individual patients following doxapram and between patient groups with differing cognitive sets.

It thus appears that breath-by-breath variation in $\mathrm{V}_{\mathrm{t}}$, quantified by the von Neumann statistic and prominently manifested as sighing, is a robust and fairly stable marker of panic disorder patients. Given the persistence of this finding across phases and instruction sets in this study, and across a variety of different experimental paradigms in other laboratories (Bystritsky and Shapiro 1992; Gorman et al 1988; Schwartz et al 1996; Stein et al 1995), it appears unlikely to be a function of the specific psychologic context to which patients are exposed. It also does not appear to be influenced by sensitivity to the specific method of respiratory monitoring, such as the mouthpiece and nose clip used in this study (Askanazi et al 1980). Its persistence suggests a fairly basic, intrinsic dysregulation of respiratory patterns in panic patients and supports interest in respiratory phenomena as a productive focus in the search for the pathophysiology of panic disorder. Further work to identify the triggers, function, and neural control of sighing patterns of breathing may well provide substantial insight into the psychobiology of panic. A first step will be to determine the specificity of this breathing abnormality for panic. Recent work supports such specificity. One study showed slow respiratory recovery in panic patients following paced, rapid breathing, accompanied by excessive sighing in the panic patients relative to both healthy control subjects and patients with social anxiety disorder (Wilhelm et al, in press). Another report showed increased $V_{t}$ instability in panic patients relative to patients with generalized anxiety disorder, and this was at least partially related to excessive sighing (Wilhelm et al 2001b).

Limitations of our study include the nonstandard definition of "sighs" that we have used and the post hoc nature of this definition and the sigh analyses. The existing literature on sighing patterns of breathing has most often defined sighs as breaths that are twice the size of a given individual's average, resting $V_{t}$. Such a definition would not be useful in the context of our study for two reasons. One, we were artificially driving $\mathrm{V}_{\mathrm{t}} \mathrm{s}$ up using doxapram, and any definition based on breath size relative to a resting average would count almost all postdoxapram breaths as sighs. A definition based on a rolling average of recent breaths was also untenable given the nature of the continuous change induced by doxapram and recovery from it. Two, we had no psychologically inert, "basal" period in which to collect resting measures. When monitoring began subjects had received a cognitive intervention that could already be affecting breathing patterns. It did in fact appear to be impacting hypothalamic-pituitary-adrenal activity (Abelson et al 1996b) and symptom ratings (Abelson et al 1996a) even during the initial accommodation period. The accommodation period also exposed subjects to the novel experience of breathing through a mouthpiece in an unusual environment. Patients, control subjects, and subjects with and without exposure to the cognitive intervention could be expected to react differently to these conditions. Associated respiratory differences could either undermine or falsely enhance group differences in sighing if the sigh definition used a purported measure of "basal" $V_{t}$ that was in fact influenced by psychologic factors that differed between groups. The groups, however, could in fact also differ in true basal $V_{t}$ in ways that might contribute to group differences in sighs using the definition employed here. However, our best available measure of basal $V_{t}$ did not show any group differences. Reanalysis of accommodation phase data using an alternative sigh definition that adjusted for 
differences in basal $\mathrm{V}_{\mathrm{t}}$ did not alter the results. It therefore appears unlikely that the finding of excessive sighing in panic patients could be due to bias introduced by the definition used.

The sigh definition we chose was based on inspection of breathing records and was an attempt to capture quantitatively what was evident there. Visual inspection suggested strongly that, regardless of "basal" $\mathrm{V}_{\mathrm{t}} \mathrm{s}$ and changing volumes with doxapram, the breathing pattern of patients was characterized by periodic, isolated breaths of considerably larger volume than the surrounding breaths. To us, this is the essence of a sigh and this is what we captured in our definition. Given that we then statistically analyzed a variable whose definition was derived from inspection of the data being analyzed, these analyses have to be considered post hoc and preliminary. But we believe they have captured something important about respiratory patterns in anxious patients. Further studies will be needed, using this and other experimental paradigms, before we can conclude that increased sighing, by this definition, is characteristic of patients with panic disorder.

If so, then more data are also needed to determine whether frequent sighs in panic patients originate in a brain stem suffocation alarm center or elsewhere in the brain. Their persistence in our data, despite the substantial impact of the cognitive intervention on respiratory and symptom measures (Abelson et al 1996a), does argue against the idea that they are epiphenomena of catastrophic misinterpretation of somatic cues. However, the stability of sigh frequency, in the face of the changes in $\mathrm{V}_{\mathrm{E}}$ and $\mathrm{pCO}_{2}$ accompanying doxapram-induced hyperventilation, also argues against a $\mathrm{pCO}_{2}$-dependent mechanism as the sigh trigger and thereby against an origin in a $\mathrm{pCO}_{2}-$ sensitive brain stem suffocation alarm center. If panic patients have a supersensitive suffocation alarm, and sighs provide protection from triggering it by reducing $\mathrm{pCO}_{2}$ below the alarm threshold (Klein 1993; Schwartz et al 1996), we should see increased sighing in patients, but this should be less evident when $\mathrm{pCO}_{2}$ is exogenously driven to low levels, as it was by doxapram. This was clearly not the case in our study. It is possible that doxapram itself provides exogenous drive to sigh mechanisms, which might obscure potential reductions in sigh rates associated with lowered $\mathrm{pCO}_{2}$. However, if this were the case we would have to invoke two different mechanisms to explain increased sighing in patients both before and after doxapram. Further work is needed to clarify this issue, but our data do not appear to us to support the hypothesis that panic patients are using sighs to keep $\mathrm{pCO}_{2}$ below a suffocation alarm threshold. They also do not support the hypothesis that they are epiphenomena of abnormal cognitive processing of somatic cues.

Although brain stem respiratory control centers can maintain the basic respiratory rhythm without input from the CNS above the medulla (Harper et al 1998), full control of respiration is quite complex and involves inputs from all levels of the brain. There is evidence that the less rhythmic, momentary changes in breathing patterns, such as those induced by affect, may be influenced by higher brain areas such as the hippocampus (Harper et al 1998). The dorsal hippocampus in fact is activated before sighs (Poe et al 1996), and the paraventricular hypothalamus may play a role in the progression of sigh/apnea breathing sequences (Kristensen et al 1997). The central nucleus of the amydgala also plays a role in respiratory control, perhaps linking alterations in respiratory patterns to conditioned cues (Zhang et al 1986). These limbic structures are all critical components of the brain's fear circuit (Gorman et al 2000), and it is certainly possible that the clear link between panic disorder and respiratory dysregulation could occur at the midbrain level. Further basic and clinical study of the role of these limbic structures in both anxiety and sighing breathing patterns should be pursued. Very recent psychophysiologic work with panic patients supports the hypothesis that sighs may be centrally triggered events and suggests that resting state hyperventilation in panic patients is primarily attributable to their excessive sighing (Wilhelm et al 2001a).

The accumulating data suggest that a deeper understanding of sighs may enhance panic research. Sighs are essential in maintaining normal lung volumes. Lung volumes decrease in the absence of sighs due to reduced compliance (the lungs become stiffer), which increases the work of breathing and can impact oxygenation. Patients under general anesthesia who are ventilated with normal, unvarying $\mathrm{V}_{\mathrm{t}} \mathrm{s}$ show significant reductions in compliance and oxygen tension, both of which are reversed by a few deep breaths (Bendixen et al 1963). In normal individuals, deep breaths can increase lung volume 26-40\% (Ferris and Pollard 1960). The presence or absence of sighing can also increase or decrease dyspnea. In normal subjects, voluntary constraint of $\mathrm{V}_{\mathrm{t}}$ produces dyspnea even in the absence of $\mathrm{pCO}_{2}$ change (Chonan et al 1987). A study of ventilated quadriplegics has also shown that subjective "air hunger" is inversely related to breath size, independently of $\mathrm{pCO}_{2}$, suggesting that mechanoreceptor information from stretch receptors in the lungs and airways is sufficient to alter sensations of dyspnea (Manning et al 1992). This effect is thought to be mediated by the vagus nerve and intermediary neurons in the midbrain and thalamus (Manning et al 1992). Breath-holding dyspnea is immediately and completely abolished by taking a deep breath, even if the inhaled gas has elevated $\mathrm{CO}_{2}$ and reduced oxygen (Fowler 1954). The impact of varying $\mathrm{V}_{\mathrm{t}} \mathrm{s}$ on lung compliance and dyspnea appear to be independent of any chemoreceptor effect. 
The above evidence supports the following hypotheses: 1) sighs are centrally triggered events, perhaps controlled by midbrain structures; 2) they account for much of the hyperventilation seen in panic patients; 3) their normal function is to increase lung compliance and reduce subjective dyspnea; and 4) these latter effects are independent of $\mathrm{pCO}_{2}$ and chemoreceptor activity. On the basis of these hypotheses we propose that the "suffocation alarm" that persistently misfires in panic patients is not brain stem based and $\mathrm{pCO}_{2}$ dependent. It may involve higher centers and a chronic vulnerability to dyspnea that is not chemoreceptor driven. Abnormal activity in limbic fear circuitry may be able to generate dyspnea, and the frequent sighing and associated hyperventilation seen in panic patients may be a compensatory response, attempting to enlist mechanoreceptors in the lungs, airways, and chest wall in chronic efforts to reduce the distressing sensation of air hunger. Follow-up work is needed to test this hypothesis. Readers can put its plausibility to a quick subjective test by taking a large $\mathrm{V}_{\mathrm{t}}$ breath, with a slow, prolonged exhalation (doing this against a partially closed upper airway produces the sound of the classic sigh). For most, a comfortable respiratory pause will follow and will be accompanied by a sense of calm.

This research was supported in part by a grant from the National Institute of Mental Health (No. R29 MH52724) and a Clinical Research Center Grant from the National Institute of Health (No. M01RR00042).

The authors thank Donald F. Klein, M.D. and Frank H. Wilhelm, Ph.D. for extremely helpful reviews of an earlier draft of the manuscript. Data processing and analyses could not have been completed without the diligent and generous work of Hedieh Briggs, M.S.W.

\section{References}

Abelson JL, Nesse RM, Weg JG, Curtis GC (1996a): Respiratory physiology and anxiety: Cognitive intervention in the doxapram model of panic. Psychosom Med 58:302-313.

Abelson JL, Weg JG, Nesse RM, Curtis GC (1996b): Neuroendocrine responses to laboratory panic: Cognitive intervention in the doxapram model. Psychoneuroendocrinology 21:375390.

Albus M, Zahn TP, Breier A (1992): Anxiogenic properties of yohimbine: II. Influence of experimental set and setting. Eur Arch Psychiatry Clin Neurosci 241:345-351.

Askanazi J, Silverberg PA, Foster RJ, Hyman AI, Milic-Emili J, Kinney JM (1980): Effects of respiratory apparatus on breathing pattern. J Appl Physiol 48:577-580.

Bendixen HH, Hedley-White J, Laver MB (1963): Impaired oxygenation in surgical patients during general anesthesia with controlled ventilation. A concept of atelectasis. $N$ Engl J Med 269:991-996.

Bystritsky A, Shapiro D (1992): Continuous physiological changes and subjective reports in panic patients: A preliminary methodological report. Biol Psychiatry 32:766-777.

Chonan T, Mulholland MB, Cherniack NS, Altose MD (1987): Effects of voluntary constraining of thoracic displacement during hypercapnia. J Appl Physiol 63:1822-1828.

Clark DM (1993): Cognitive mediation of panic attacks induced by biological challenge tests. Adv Behav Res Ther 15:75-84.

Ferris BG, Pollard DS (1960): Effect of deep and quiet breathing on pulmonary compliance in man. J Clin Invest 39:143-149.

Fowler WS (1954): Breaking point of breath-holding. J Appl Physiol 6:539-545.

Gorman JM, Fyer MR, Goetz R, Askanazi J, Liebowitz MR, Fyer AJ, et al (1988): Ventilatory physiology of patients with panic disorder. Arch Gen Psychiatry 45:31-39.

Gorman JM, Kent JM, Sullivan GM, Coplan JD (2000): Neuroanatomical hypothesis of panic disorder, revised. Am J Psychiatry 157:493-505.

Harper RM, Poe GR, Rector DM, Kristensen MP (1998): Relationships between hippocampal activity and breathing patterns. Neurosci Biobehav Rev 22:233-236.

Klein DF (1993): False suffocation alarms, spontaneous panics, and related conditions: An integrative hypothesis. Arch Gen Psychiatry 50:306-317.

Kristensen MP, Poe GR, Rector DM, Harper RM (1997): Activity changes of the cat paraventricular hypothalamus during phasic respiratory events. Neuroscience 80:811-819.

Lee YJ, Curtis GC, Weg JG, Abelson JL, Modell JG, Campbell K (1993): Panic attacks induced by doxapram. Biol Psychiatry 33:295-297.

Manning HL, Shea SA, Schwartzstein RM, Lansing RW, Brown R, Banzett RB (1992): Reduced tidal volume increases "air hunger" at fixed $\mathrm{PCO} 2$ in ventilated quadriplegics. Respir Physiol 90:19-30.

Papp LA, Klein DF, Gorman JM (1993): Carbon dioxide hypersensitivity, hyperventilation, and panic disorder. Am J Psychiatry 150:1149-1157.

Papp LA, Martinez JM, Klein DF, Coplan JD, Norman RG, Cole R, et al (1997): Respiratory psychophysiology of panic disorder: Three respiratory challenges in 98 subjects. Am $J$ Psychiatry 154:1557-1565.

Poe GR, Kristensen MP, Rector DM, Harper RM (1996): Hippocampal activity during transient respiratory events in the freely behaving cat. Neuroscience 72:39-48.

Rapee RM, Mattick R, Murrell E (1986): Cognitive mediation in the affective component of spontaneous panic attacks. $J$ Behav Ther Exp Psychiatry 17:245-253.

Roth WT, Wilhelm FH, Trabert W (1998): Voluntary breath holding in panic and generalized anxiety disorder. Psychosom Med 60:671-679.

Sanderson WC, Rapee RM, Barlow DH (1989): The influence of an illusion of control on panic attacks induced via inhalation of 5.5\% carbon dioxide enriched air. Arch Gen Psychiatry $46: 157-162$

Schwartz GE, Goetz RR, Klein DF, Endicott J, Gorman JM (1996): Tidal volume of respiration and "sighing" as indicators of breathing irregularities in panic disorder patients. Anxiety 2:145-148. 
Stein MB, Millar TW, Larsen DK, Kryger MH (1995): Irregular breathing patterns during sleep in patients with panic disorder. Am J Psychiatry 152:1168-1173.

van der Molen GM, van den Hout MA, Vroemen J, Lousberg H, Griez E (1986): Cognitive determinants of lactate-induced anxiety. Behav Res Ther 24:677-680.

Wilhelm FH, Gerlach AL, Roth WT (in press): Slow recovery from voluntary hyperventilation in panic disorder. Psychosom Med.
Wilhelm FH, Trabert W, Roth WT (2001a): Characteristics of sighing in panic disorder. Biol Psychiatry 49:606-614.

Wilhelm FH, Trabert W, Roth WT (2001b): Physiological instability in panic disorder and generalized anxiety disorder. Biol Psychiatry 49:596-605.

Zhang JX, Harper RM, Ni HF (1986): Cryogenic blockade of the central nucleus of the amygdala attenuates aversively conditioned blood pressure and respiratory responses. Brain Res $386: 136-145$ 